Ebisu Ebisu

Études japonaises Études japonaises

47 | printemps-été 2012

Catastrophes du 11 mars 2011, désastre de

Fukushima : fractures et émergences

\title{
Les voix des femmes après la catastrophe du 11
}

\section{mars}

Voices of Women After March 11th

3・11東日本大震咨の女性たちの声

\section{Christine Lévy}

\section{OpenEdition}

\section{Journals}

Édition électronique

URL : http://journals.openedition.org/ebisu/363

DOI : 10.4000/ebisu.363

ISSN : 2189-1893

Éditeur :

Institut français de recherche sur le Japon (UMIFRE 19 MAEE-CNRS), Maison franco-japonaise

Édition imprimée

Date de publication : 1 juin 2012

Pagination : 133-141

ISSN : 1340-3656

Référence électronique

Christine Lévy, «Les voix des femmes après la catastrophe du 11 mars », Ebisu [En ligne], 47 | printemps-été 2012, mis en ligne le 17 juin 2014, consulté le 30 avril 2019. URL : http:// journals.openedition.org/ebisu/363 ; DOI : 10.4000/ebisu.363 


\title{
Les voix des femmes après la catastrophe du 11 mars
}

\author{
Christine LÉVY
}

Quels sont les problèmes spécifiquement liés au genre parmi les conséquences immédiates et à moyen terme de la catastrophe du 11 mars? Quels défis doivent relever celles ou ceux qui mènent une lutte contre les discriminations liées au genre ? Ces questions rejoignent les problématiques soulevées par des militantes comme Naomi Klein (2007) ou Rebecca Solnit (2006) ${ }^{1}$ : le pire (Klein) comme le meilleur (Solnit) peut naître d'une catastrophe qui touche les populations dans leurs chairs et au plus profond de leurs cœurs. Les images de détresse, de deuil impossible, transmises par la télévision comme par la presse écrite ont fortement contribué à créer une "communauté de sympathie » (kyōkan no kyödōtai 共感の共同体), indispensable pour recréer les liens de solidarité dont a besoin le tissu social pour se reconstituer, mais qui peut aussi servir, si l'on n'y prend garde, à la résurgence et au primat de la "communauté nationale ", comme le souligne Sakai Naoki 酒井直樹 (2011). Prolongeant les analyses proposées par Maruyama Masao 丸山真男 (1946) en son temps sur le fascisme et le militarisme japonais,

\footnotetext{
\ Christine Lévy, actuellement chercheure à la Maison franco-japonaise de Tokyo (UMIFRE 19 CNRS-MAEE), est maître de conférences à l'université de Bordeaux 3. Elle travaille sur l'histoire des idées politiques et sur le genre dans le Japon moderne et contemporain.

1. En son temps Kōtoku Shūsui 幸徳秋水 (1871-1911), réfugié alors aux États-Unis, avait relaté son expérience du tremblement de terre de San Francisco en 1906. Il fut convaincu de la capacité des gens à s'organiser par eux-mêmes et renforcé dans ses convictions politiques anarchistes.
} 
Sakai Naoki met en garde contre la tendance des médias à participer à ce système d'irresponsabilité qui, en privilégiant la construction d'une communauté de consolation réciproque, détourne le public de la question de la responsabilité des industriels, de l'administration, des hommes politiques et des collectivités locales. De plus, en relayant unanimement les consignes de jishuku 自肃 (retenue) - une notion certes légitime pour exprimer le deuil de toute une nation -, ces médias ont contribué à faire taire les voix ou les actions discordantes, surtout celles qui demandent des comptes (Sakai $2011: 28$ ).

Il n'en reste pas moins que c'est une société tout entière qui est blessée et le traumatisme d'une telle catastrophe ne saurait se circonscrire à la seule région du Nord-Est, frappée de plein fouet. Chacun peut se souvenir d'une image qui l'aura plus particulièrement ému et bouleversé. Le traumatisme s'étend ainsi à ceux qui n'ont pas été directement victimes et l'augmentation très importante du nombre de suicides dans les grandes villes comme Osaka et Tokyo depuis mai 2011 (Kayama 2011 : 24) en constitue peutêtre un symptôme. Les sentiments d'impuissance, de culpabilité peuvent aussi nourrir des conflits au sein des couples ou encore sur les lieux de travail, et provoquer des ruptures (Kayama 2011 : 25). Dans un tel contexte, la réminiscence de la dernière guerre - le mensonge d'État sur la situation militaire est comparé au mensonge sur la sécurité nucléaire, les morts provoquées par le tsunami aux soldats sacrifiés - montre à quel point le lien entre les morts et les vivants - terrain privilégié de la religion - est bouleversé. Dans une société sécularisée, la religion ne suffit plus à combler les angoisses face à la mort, et l'identification à un destin national permet de glorifier ou de donner sens à ce qui autrement n'apparaîtrait que comme une absurdité insupportable. Mais dans le quotidien de la survie, de nouveaux rapports d'entraide apportent aux sinistrés plongés dans le désespoir, un réconfort et parfois une lueur d'espoir. Les organisations de soutien qui se sont formées pour fournir une aide matérielle et psychologique jouent un rôle important dans cette perspective, s'appuyant sur les enseignements tirés de l'expérience de l'après séisme de Kobe en 1995 (Hanshin Awaji daishinsai 阪神・淡路大震災). L'élan de solidarité surgi après ce séisme de 1995 fut le point de départ de deux notions nouvelles au Japon "vorantia» ヴオランティア (bénévolat) et "kokoro no kea»こころのケア (soins psychologiques) (Nakai 2011 : 56). Les femmes y furent particulièrement impliquées ; ainsi, lorsque le département de Hyōgo (celle où se trouve la ville de Kobe) 
décida de créer un centre dédié au soutien psychologique (et pour lequel fut voté un budget quinquennal), les divers groupes qui en avaient été à l'initiative et le composaient étaient constitués à plus de $90 \%$ de femmes (Nakai 2011 : 56). C'est dire si des organisations féminines ou féministes se sont mobilisées pour joindre leurs forces et créer des réseaux de soutiens concrets. Mais aujourd'hui leur constat est contrasté. Elles insistent davantage sur la nécessité de créer un terrain propice à l'expression de la volonté des femmes, et de faire entendre d'autres voix que celles qui se figent dans un faux unanimisme où il ne serait plus question que de s'unir et de faire taire des voix « égoïstes » ou dissonantes.

Car de ces alternatives qui auraient pu surgir après la catastrophe, celle qui domine est une tendance à la répétition et à la reconduction du même : les femmes ont eu des difficultés à se faire entendre, à exister en tant que telles ou à porter un point de vue ou des revendications spécifiques. Il leur est encore plus difficile d'être présentes dans les instances de décision ou de réflexion sur la reconstruction. Les femmes sont quasi absentes des divers organismes de réflexion mis en place par le gouvernement ou par les autorités locales : le Higashi Nihon daishinsai fukkō kōsō kaigi 東日本大震災復興 構想会議 (Conseil pour les projets de reconstruction après le grand séisme de l'Est du Japon $)^{2}$ ne compte qu'une seule femme parmi ses quinze membres, et dans son comité consultatif, deux femmes sur dix-neuf membres. Il en va de même pour les instances régionales, par exemple dans le département de Kanagawa, le comité composé de 56 membres compte une seule femme, maire de la ville de Yokohama. On a pu constater aussi, concrètement, dans les centres de refuge, la reconduction de la division sexuelle des tâches, et son renforcement dans l'organisation de la survie élémentaire. La division des tâches selon le genre a eu tendance à s'accentuer en ces périodes difficiles, les femmes étant systématiquement assignées aux tâches dites « domestiques » comme la préparation des repas, le soin aux personnes âgées et aux enfants, et de ce fait exclues des tâches d'organisation. Cette tendance est assez manifeste et des articles qui relatent les exemples les plus réussis de l'organisation de la vie dans les centres de refuge soulignent la présence des hommes plutôt jeunes, de vingt à quarante ans : l'organisation exemplaire du centre de refuge dans le premier collège municipal de Rikuzentakada

2. Son site officiel est : <http://www.cas.go.jp/jp/fukkou/>. 
陸前高田, une des zones les plus touchées dans le département d'Iwate (Mihashi 2011) et qui a attiré les journalistes du monde entier, ne déroge pas à cette règle ${ }^{3}$.

C'est en réponse à cette situation que fut créé le réseau Rise Together for Women in East Japan Desaster ${ }^{4}$. Lors de la conférence de presse du 24 mai 2011, une de ses représentantes, Takenobu Mieko 竹信三恵子, insistait surtout sur l'importance de créer dans un contexte de prolongation de la durée du séjour dans les centres d'évacuation, une atmosphère où il est possible de s'exprimer, où il est permis de " dire les choses ". Elle déclarait que si les femmes ont des besoins spécifiques, il est important de les faire entendre sans les faire passer pour des exigences égoïstes. Créer des méthodes pour les reconnaître et les entendre, tel est le but que se donne ce réseau. Masai Reiko 正井礼子, du même réseau, explique par exemple, qu'à la suite du séisme de Kobe, lorsqu'elle a interrogé les femmes sur ce qu'elles désiraient le plus, celles-ci ont répondu : "du travail plutôt que le care ». Certes, il faut entendre une double inquiétude dans cette réponse. Car, comme l'a constaté une psychologue clinicienne dans ses interventions après le 11 mars (Ōsawa 2011 : 48), le refus du care psychologique est le plus souvent dicté par la crainte de passer pour une personne à problèmes au regard des autres. Cette attitude nous rappelle combien la communauté locale et traditionnelle, si elle est pourvoyeuse de soutien et constitue un lieu d'entraide, exige aussi de ses membres le respect d'une identité commune et des frontières qu'elle a elle-même délimitées. ${ }^{5}$. Mais ces femmes exprimaient avant tout leur préoccupation fondamentale, l'idée que sans moyen de vivre elles ne pouvaient se projeter dans le futur. Leur réponse est

3. Certes, ceci nuance l'optimisme de l'idée défendue par Rebecca Solnit d'une naissance d'une communauté de type utopique. Néanmoins le succès de son ouvrage auprès de nombreux lecteurs japonais indique qu'ils y ont puisé leur inspiration pour agir en tant qu'individus en solidarité avec d'autres individus, loin de tomber dans le cliché de l'exaltation du caractère national.

4. Le nom japonais est Higashi Nihon dai shinsai - Josei shien nettowāku 東日本大震 災・女性支援ネットワーク : <http://www.risetogetherjp.org/>.

5. La répression politique au Japon s'est beaucoup appuyée sur ce système depuis l'époque Edo, d'une part en ghettö̈sant systématiquement les burakumin et d'autre part en créant un système de solidarité dans chaque communauté villageoise. Ce mécanisme a continué à fonctionner à l'ère Meiji et a été renforcé lors de la montée du militarisme. 
très claire et très importante pour rappeler que les personnes sinistrées ont besoin de soutien non pas seulement ponctuel, mais d'une infrastructure qui leur offre la possibilité de se projeter à long terme pour reconstruire leur vie. Comment cela est-il envisageable?

Dans un contexte de crise et de détérioration des services publics depuis des années, l'augmentation de la pauvreté, la déréglementation du travail (qui toutes deux touchent en premier les femmes), viennent aggraver les difficultés des sinistrés à retrouver un emploi, en particulier pour les femmes $^{6}$. Rappelons que $60 \%$ des travailleurs employés par les entreprises de sous-traitance pour la centrale nucléaire de Fukushima sont des personnes sinistrées ${ }^{7}$. L'environnement est de plus menacé de façon dramatique dans le département de Fukushima, dont les habitants sont obligés de se poser tous les jours la question du degré de contamination radioactive des aliments, s'ils ont raison ou tort de rester sur place ou comment fuir cette situation. Les mois passent et les questions angoissantes s'accumulent sans trouver de réponse ni de solution. Les personnes et les familles dont le lieu d'habitation n'est pas intégré dans la zone d'évacuation désignée par le gouvernement souffrent de l'indifférence. Les tentatives du gouvernement et en particulier du ministère de la Santé et du Travail (Kōrōshō 厚労省) pour rassurer les mères, par exemple par l'édition d'une brochure tirée à trois millions d'exemplaires, en avril 2011, contrastent de façon saisissante avec d'autres brochures élaborées en direction du même public ${ }^{8}$. Alors que cellesci ont pour but d'attirer l'attention sur tous les dangers potentiels comme la listéria ou autres produits toxiques (comme le mercure méthylique contenu

6. Dans les trois départements d'Iwate, de Miyagi et de Fukushima, 37601 femmes contre 26631 hommes touchent des allocations de chômage en novembre 2011, selon une enquête publiée (23 janvier 2012) par le Kōrōshō. Avant le 11 mars la proportion était égale entre hommes et femmes, ce qui montre bien que les femmes sont davantage touchées par le chômage depuis le désastre. Cela tient d'une part au fait que les secteurs liés à la reconstruction emploient plus d'hommes et aux difficultés de reprise des secteurs industriels sinistrés.

7. Voir l'article de Paul Jobin dans ce même numéro.

8. Voir la liste de brochures : <http://www.mhlw.go.jp/topics/syokuchu/06.html>. 
dans certains poissons ${ }^{9}$ ), la brochure éditée ${ }^{10}$ sur la question de la contamination radioactive révèle par l'expression choisie dans le titre, anshin 安心 (la tranquillité, être rassuré) qu'il s’agit davantage de rassurer que de garantir la sécurité alimentaire. Subrepticement l'expression anshin remplace anzen 安全 (la sécurité). Pour les mères qui vivent à Fukushima et qui sont confrontées en permanence à la question de la contamination, cela n'a rien de rassurant, bien au contraire. Entendre les représentants du gouvernement, de Tepco et certains scientifiques (comme Yamashita Shun.ichi 山下俊一 $)^{11}$ leur affirmer que l'eau, l'air et la nourriture sont sains et sans danger les plongent dans la colère et les poussent à agir.

Lorsque le gouvernement décida de relever le niveau d'admissibilité du taux d'exposition aux radiations de 1 à $20 \mathrm{mSv}$ par an ${ }^{12}$ pour maintenir l'ouverture des écoles, le "Réseau de Fukushima pour la protection des enfants contre les radiations ${ }^{13}$ " fut mis en place le $1^{\text {er }}$ mai, autour de mères de famille ${ }^{14}$; depuis, le réseau s'est organisé et étoffé. Le groupe s'est formé avec les habitants qui voulaient mesurer par eux-mêmes les niveaux de radiation, dépolluer si possible, et aider à évacuer le cas échéant. Mais devant la dangerosité de la réalité, l'organisation a commencé à insister davantage sur la nécessité d'évacuer les enfants et d'aider les familles à le faire. Le point d'orgue de cette prise en main de leur propre sort a été l'organisation du sit-in devant les bâtiments du METI, le ministère de l'Économie, du Commerce et de l'Industrie, les 27, 28 et 29 octobre. Ces trois journées ont permis de sensibiliser et de rappeler les problèmes de santé auxquels la population doit faire face, les cent femmes venues de Fukushima ont

9. Source: <http://www.mhlw.go.jp/topics/bukyoku/iyaku/syoku-anzen/suigin/index. html> (consulté le 28 novembre 2011).

10. Source : <http://www.mhlw.go.jp/stf/houdou/2r98520000014hcd-img/2r985200 00014hdu.pdf> (consulté le 28 novembre 2011).

11. Voir l'article de Paul Jobin dans ce même numéro.

12. C'est la limite autorisée pour les personnels exposés, en France (Code du travail, Article R231-76). La limite autorisée pour l'exposition de la population aux rayonnements artificiels, en France est de $1 \mathrm{mSv} / \mathrm{an} /$ personne (Code de la santé publique, Article R1333-8).

13. Kodomotachi o hōshanō kara mamoru Fukushima nettowāku 子どもたちを放射能か ら守る福島ネットワーク.

14. Voir : <http://fukushima.over-blog.fr/article-100-femmes-pour-sauver-les-enfantsde-fukushima-87701324.html> (consulté le 17 novembre 2011). 
demandé une audience auprès de représentants du METI pour présenter les quatre revendications suivantes : 1) l'arrêt immédiat des centrales ; 2) le non redémarrage de celles en cours de test ; 3) l'évacuation de tous les enfants sous la responsabilité de l'État et la prise en charge financière des frais occasionnés, y compris pour ceux qui sont déjà partis ; et 4) la suppres-

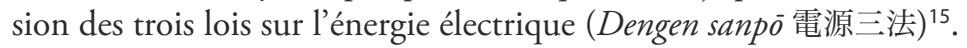

Dans leur mouvement, elles ne se cantonnent pas à vouloir protéger leurs enfants, mais cherchent à comprendre les causes qui ont rendu possible cet état de fait et à en sortir. Affirmer la nécessité de remplacer le nucléaire par des ressources énergétiques compatibles avec la vie, c’est aussi renouer avec le mouvement antinucléaire qui avait été très fort à partir des années 1950 et ce jusque dans les années 1970. Elles ont pris conscience du recul de ce mouvement face à l'immense pouvoir d'argent du lobby nucléaire ${ }^{16}$, et des conséquences actuelles. Dans les années 1950, le grand mouvement lancé contre les expérimentations de la bombe $\mathrm{H}$ avait pour composante essentielle le mouvement des mères, le hahaoya undō 母親運動. La figure de la mère est-elle en passe de (re)devenir celle du citoyen ordinaire, non expert, exclu des décisions qui le concernent directement, et qui refusant cet état de fait, s'organise pour exiger sa participation aux décisions cruciales, comme le suggère, par exemple, Mizushima Nozomi 水島希 (2011: 23)? Ceci pourrait renvoyer au stéréotype de la femme qui serait plus proche de la nature et qui agirait davantage sous le coup de l'émotion que de la raison.

15. Ces trois lois sont : 1) Dengen kaihatsu sokushin zeihō 電源開発促進税法 (Loi sur la taxe pour promouvoir le développement des ressources énergétiques) adoptée en 1974 après le choc pétrolier. Chaque entreprise productrice d'électricité paye cette taxe de 375 yen pour 1000 kwh vendus ; 2) Tokubetsu kaikei ni kansuru hōritsu 特別会計に関 する法律 (Loi sur les comptabilités exceptionnelles) qui a intégré en 2007 l'ancienne loi sur la comptabilité des ressources énergétiques ; 3) Hatsuden yō shisetsu shühen chiiki seibi hō 発電用施設周辺地域整備法 (Loi sur l'aménagement des zones riveraines aux équipements destinés à la production électrique). D’après un article du Tökyō shinbun 東京新 聞 du 30 septembre 2011, $51 \%$ de cette taxe est versé aux organismes de recherches ou aux entreprises créés sous la tutelle du METI (Kiriyama 2011). Dans ces 9 organismes on retrouve 26 anciens hauts fonctionnaires (amakudari 天下り) dont quatre dirigent la Japan Atomic Energy Agency (JAEA) (Nihon genshiryoku kenkyū kaihatsu kikō 日本原 子力研究開発機構) ainsi que quatre autres la Japan Nuclear Energy Safety Organization (JNES) (Genshiryoku anzen kiban kikō 原子力安全基盤機構), sous la tutelle du METI. 16. Voir le témoignage de Kamata Satoshi 鎌田慧 (2011) traduit dans ce numéro. 
Contre cette image simplificatrice, il faut rappeler que leurs revendications sont aussi celles de leurs maris et de leurs fils qui, pris au piège du chantage à l'emploi ne peuvent manifester aussi ouvertement qu'elles le font. Ce que les femmes de Fukushima rappellent aux représentants des ministères et aux membres du gouvernement ${ }^{17}$, c'est leur incapacité à prévoir et à empêcher un prochain accident dont le coût signifie accepter le sacrifice de la vie d'une minorité : c'est le refus de cette rationalité déshumanisée et destructrice qu'elles ont décidé de faire entendre.

10 décembre 2012

\section{Bibliographie}

\section{KAMATA Satoshi 鎌田慧, 2011}

«Kyozetsu kara rentai e» 拒絶から連帯へ

(Du refus à la solidarité), Gendai shisō

現代思想, octobre, 39 (14) : 34-45 [trad.

française dans ce présent numéro].

KAYAMA Rika 香山リカ, 2011

«"Hisaichi gai" ni tsuite kangaeru »

「被災地外」について考える

(Penser les «zones extérieures » aux zones sinistrées), Gendai shisō, supplément Imago du mois de septembre, 39 (12) : 21-25.

\section{KLEIN Naomi, 2007}

The Shock Doctrine: The Rise of Disaster Capitalism, New York, Metropolitan Books.

MARUYAMA Masao 丸山真男, 1946 "Chōkokkashugi no ronri to shinri » 超国家主義の論理と心理 (Théorie et psychologie de l'ultranationalisme), Sekai 世界, mai [repris : 1956, in Gendai seiji no shisō to kōdō 現代政治の思想 と行動 (Pensées et actions politiques contemporaines), vol. 1, Miraisha

17. Voir : <http://www.ustream.tv/recorded/18140229\#utm_campaign=synclickback \&source=http://www.risetogetherjp.org/\&medium=18140229> (consulté le 30 octobre 2011). 
未來社 ; trad. anglaise : 1963, Ivan MORRIS (ed.), Thought and Behaviour in Modern Japanese Politics, London, New York, Oxford University Press.

MITSUHASHI Asako 三橋麻子, 2011 « Hinanjo de rīdā ni natta » 避難所で リーダーになった (Leader dans les centres de réfugiés), Aera アエラ, 2-9 mai, $21: 29-32$.

\section{MIZUSHIMA Nozomi 水島希, 2011} «Gijutsu to chi o watashitachi no te ni torimodosu »技術と知をわたしたちの手 にとりもどす (Reprenons en main la technique et le savoir), Onna tachi no nijūisseiki 女たちの21世紀 (Le $21^{\mathrm{e}}$ siècle des femmes), AJWRC (Asian Japan Women's Resource Center), septembre, 67 : 19-23.

\section{NAKAI Hisao 中井久夫, 2011}

«Daishinsai, PTSD, debreifing » 大震災、PTSD、ディブリフィング

(Le grand séisme, SSPT et débriefing), Gendai shisō, supplément Imago du mois de septembre, 39 (12) : 56-66.

ŌSAWA Tomoko 大澤智子, 2011 "Arayuru koto ga "kokoro no kea" ni nariuru » あらゆることが『こころの ケア』になりうる (Tout peut servir au care psychologique), Gendai shisō, supplément Imago du mois de semptembre, 39 (12) : 48-55.

SAKAI Naoki 酒井直樹, 2011

«"Musekinin no taikei" mitabi»「無責任 の体系」三たび (L'irresponsabilité érigée en système, pour la troisième fois), Gendai shisō, mai, 39 (7) : 26-33.
SOLNIT Rebecca et alii, 2006

After the Ruins, 1906 and 2006:

Rephotographing the San Francisco

Earthquake and Fire, University of

California Press [traduction japonaise :

Saigai yūtopia 災害ユートピア

(L'utopie dans le désastre), Tokyo,

Aki shobō 亜紀書房，2010).

KIRIYMA Junpei 桐山純平, 2011

«Ene tokkai no genpatsu yosan amakudari no hōjin ni hangaku chō shishutsu » エネ特会の原発予算、 天下りの法人に半額超支出 (Le budget extraordinaire alloué au nucléaire : plus de la moitié versée aux sociétés de pantouflage), Tōkyō shinbun 東京新聞, 30 septembre, pp. 1-2. 